http://www.ejchem.net 2012, 9(2), 716-728

\title{
Characterization and Assessment of Flavor Compounds and Some Allergens in Three Iranian Rice Cultivars during Gelatinization Process by HS-SPME/GC-MS
}

\author{
M. H. GIVIANRAD* \\ Department of Chemistry, Science and Research Branch, \\ Islamic Azad University, Tehran, Iran \\ givianradh@yahoo.com
}

Received 20 August 2011; Accepted 27 October 2011

\begin{abstract}
A combined gas chromatography mass spectrometry with headspace solid-phase microextraction method has been utilized for the analysis of the flavor volatiles of three different rice cultivars including two modified Iranian rice cultivars and Hashemi rice cultivar during gelatinization. As a result, while gelatinization would progress, the amount of the volatile compounds would be also increased. Altogether, 74, 55 and 66 components were identified for Hashemi, HD5 and HD6 rice samples, respectively, which 56 unique compounds were not identified, previously. Subsequently, seven fragrance chemicals have been detected, which were most frequently reported as contact allergens in the European Union.
\end{abstract}

Keywords: Rice (Oryza sativa L.), Flavor Volatiles Components, Solid-Phase Microextraction (SPME), Gas Chromatography Mass Spectrometry (GC/MS), Contact Allergens.

\section{Introduction}

Rice is the major product of people's diet in many countries and also one of the most momentous factors in market business is the aroma of 'fragrant rices'. This is a feature which discriminates it from regular rice ${ }^{1,2}$.

There are numerous volatile components in cooked rice grains, as well as those which are due to biochemically distinct pathways, and are very rich sources of hydrocarbons, organic acids, alcohols, aldehydes, ketones, esters and phenols. On the other hand, there are some other compounds which are extracted as a result of chemical breakdowns, which fatty acids are good sample among them ${ }^{3}$.

Aromatic can have both positive and negative influence on people's tastes. The former can be flavor and fragrance components such as 2-acetyl-1-pyrroline (2AP) and the aromatic alcohols and the latter can be off-flavors of hexanal and 2-pentylfuran ${ }^{4} .2 \mathrm{AP}$ is one of the 
aromatic compounds, which recognized as the most imperative key flavor component of rice $\operatorname{aroma}^{5-8}$.

In order to afford sample preparation methods and analytical techniques, Solid-phase microextraction (SPME) is introduced as a rapid, sensitive and consistent technique for the extraction and concentration of volatile compounds from different sample matrices. This technique has many advantages as well as less time for extraction, effortlessness, preventing the loss of analytes and field sampling with portable field sampler ${ }^{9,10}$.

This technique has a enormous sensitivity mostly in the study of identifying flavor indicators of rice, Since the extracted fraction on the fiber is statistically introduced into the gas chromatography (GC) by thermal desorption ${ }^{11,12}$. In present study, our main intention is to use HS-SPME-GC-MS technique as a reliable method for effective trapping and screening of a broad range volatile flavor compounds in the headspace of Iranian rice samples during the gelatinization process.

\section{Experimental}

Three Iranian fragrant rice samples were employed in this study, including two new modified varieties (HD5, HD6) and one Hashemi variety (HD1), which are consumed in Iran, predominantly. These samples were collected from Rice Research Institute. All samples were harvested in August 2009 and contained 22\% moisture. Following $24 \mathrm{~h}$ from harvesting, the samples were sun-dried to about $12-13 \%$ moisture content, after that they were dehulled at the growing area and were transferred to the laboratory and stored in nylon bags and located in the refrigerator at $4{ }^{\circ} \mathrm{C}$ until the experiments were accomplished.

\section{Gelatinization process}

Firstly, some glass balls were poured into a glass of $275 \mathrm{~mL}$ water and were placed under primary heating till they reach to the boiling point. Afterward, $50 \mathrm{~g}$ of one of the tested samples was added into the boiling water and stirred. After $7 \mathrm{~min}, 10$ rice grains were chosen randomly and placed on a glass plate with equal distance. Subsequently, the samples were covered with another plate, slip it till gelatinized grains observed and then the number of gelatinized rice grains were counted, accurately. Following $8 \mathrm{~min}$, the experiment were carried out every one minute to complete the gelatinization all of the grains. This method was used for all samples in this study, which was performed for the first time.

\section{Rice cooking}

The conventional Iranian rice cooking was utilized, by which a mixture of $150 \mathrm{~g}$ of white rice and $400 \mathrm{~mL}$ of distilled water were added. The whole process was divided into 4 steps, including all the stages from the beginning till the end of gelatinization process. The cooking time of this process was determined by the temperature at which melting of crystalline structure took place. Rice with high GT requires more time to cook, while rice with low GT, requires less time; usually up to $4 \mathrm{~min}$. The earlier has unacceptable texture ${ }^{2}$.

\section{Headspace solid-phase microextraction sampling}

Process of gelatinization was accomplished Using SPME, a sampling device was designed in order to collect the sample of flavor volatiles. The flavor volatiles of rice during gelatinization were released out throughout the first side arm, while SPME fiber was located in the second side of sampling device with a flexible septum. In all stages of gelatinization, the fiber was located in the manually operated SPME holder and the septum was covered with a Tefloncoated silicone in order to prevent volatile components release in septum. The fiber was lowered in the sampling port to adsorb flavor volatile components of rice and desorbed them thermally in the injection port of GC-MS instrument for $10 \mathrm{~min}$ at $250{ }^{\circ} \mathrm{C}$. 
Afterwards, the fiber was exposed to the SPME fiber conditioner at $250{ }^{\circ} \mathrm{C}$ for $1 \mathrm{hr}$ for reconditioning before being subjected to the next volatile samples. SPME fibers with PDMS (100 $\mu \mathrm{m}$, non-bonded), CAR/PDMS (75 $\mu \mathrm{m}$, bonded) and DVB/CAR/PDMS $(65 \mu \mathrm{m}$, bonded) coating, provided by Supelco (Bellefonte, PA, USA) were applied as commercial fibers and were preconditioned in an SPME fiber conditioner (GL Sciences) at $250{ }^{\circ} \mathrm{C}$ for $1 \mathrm{~h}$ before the first measurement.

\section{Gas chromatography-mass spectrometry}

GC separation was performed on an HP-6890 GC system (HEWLETT PACKED, USA), coupled to a mass detector (HP-5973, USA). A column, HP-5MS (5\% Phenyl dimethyl siloxan) was applied using a $30 \mathrm{~m} \times 0.25 \mathrm{~mm} \times 0.32 \mu \mathrm{m}$ film thickness. Analytes extracted onto the commercial fiber, were injected using $9.5 \mathrm{~mm}$ Thermogreen LB-2 septa (Supelco). The injector's temperature was $250^{\circ} \mathrm{C}$ in this case. Helium purified at $99.99 \%$ was selected as a carrier gas at a flow rate of $1 \mathrm{~mL} / \mathrm{min}$. The temperature program was performed at $60^{\circ} \mathrm{C}$ for $3 \mathrm{~min}$, then increased at $220^{\circ} \mathrm{C}$ at rate of $5^{\circ} \mathrm{C} / \mathrm{min}$ and maintained at $220{ }^{\circ} \mathrm{C}$. The mass selective detector was applied in an electron impact ionization mode at $70 \mathrm{ev}$.

An alkane mixture with C8-C20 alkane and concentration of $40 \mathrm{mg} / \mathrm{mL}$ in hexane was purchased from Fluka. The mixture was used to approximate retention indices (RI), which was injected into the fiber by $5 \mathrm{~min}$ headspace extraction from a $10 \mathrm{~mL}$ SPME vial, including $1 \mathrm{~mL}$ HPLC-grade water spiked with $10 \mu \mathrm{L}$ of the mixture. The volatile components were positively identified by matching their mass spectra, with the spectra of reference compounds in Adams mass spectra library (9th edition) and verified on the basis of mass spectra and RI values reported in the literature ${ }^{13,14}$.

\section{Results and Discussion}

\section{Cooking time}

The cooking time of rice is determined by the temperature at which the crystalline structure of the starch begins to melt. This is called gelatinization temperature (GT). In rice samples, GT ranges from $55-85{ }^{\circ} \mathrm{C}$. Hashemi rice sample with high GT requires more time to cook. Lowering the GT of the modified (HD5, HD6) rice grains could decrease average cooking time.

In Figures 1, 2 and 3, gelatinization time for $0 \%, 50 \%, 90 \%$ and $100 \%$ of the whole grains is recognized and during this time, SPME fiber is injected. The whole process was separated into four stages: I, 17':00; II, 21':00; III, 24':90"; IV, 27':30 were assigned to Hashemi rice sample leading to gelatinization $0,50,90$, and 100 percent of rice grains. For HD5 variety at cooking stages: I, 15':00; II, 18':42"; III, 20':69"; IV, 22':27" leading to gelatinization $0,50,90$ and 100 percent of rice grains. In HD6 variety at cooking stages: I, 15':00; II, 18':38"; III, 21':67"; IV, 24':30 leading to gelatinization 0, 50, 90 and 100 percent of rice grains. 
Characterization and Assessment of Flavor Compounds and Some 719

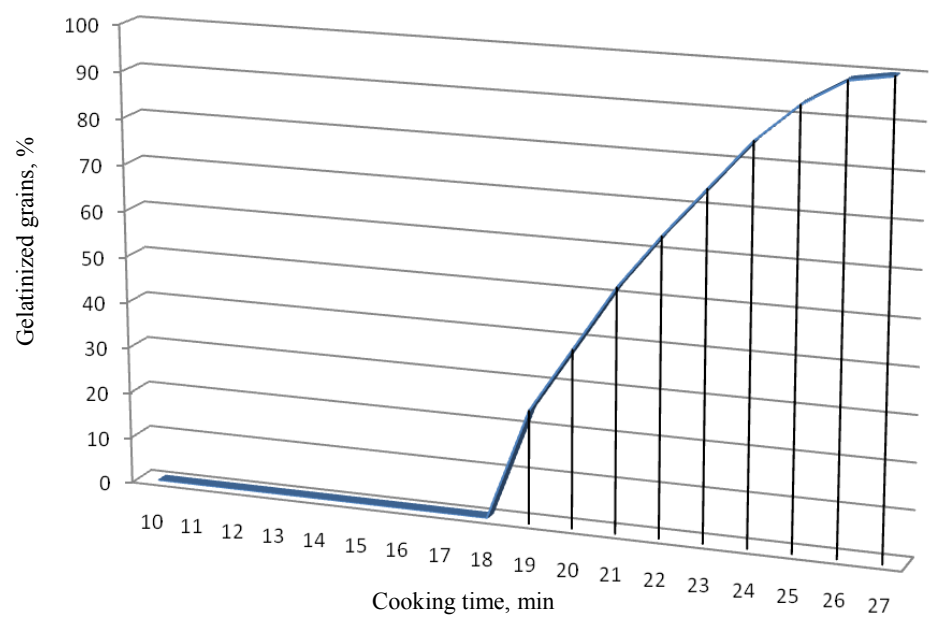

Figure 1. Gelatinization profile of Hashemi rice samples.

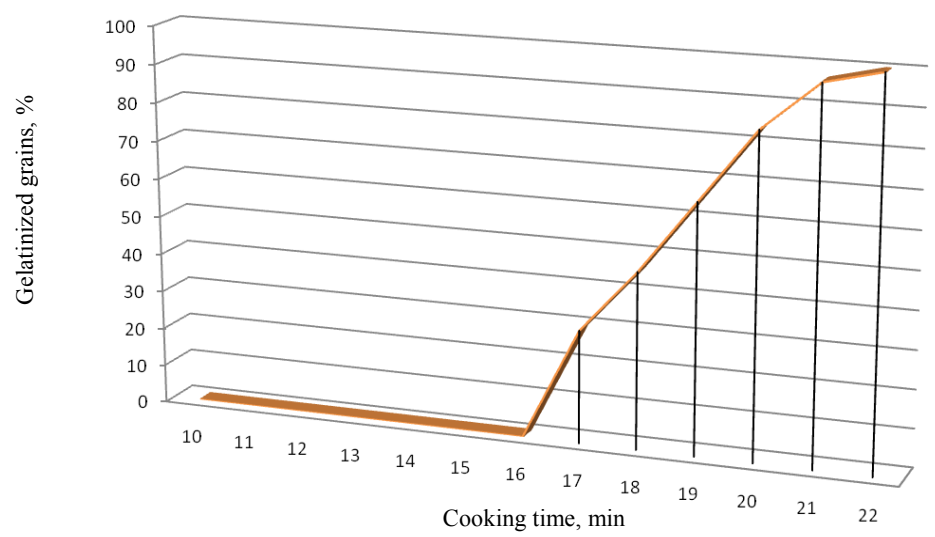

Figure 2. Gelatinization profile of HD5 rice samples.

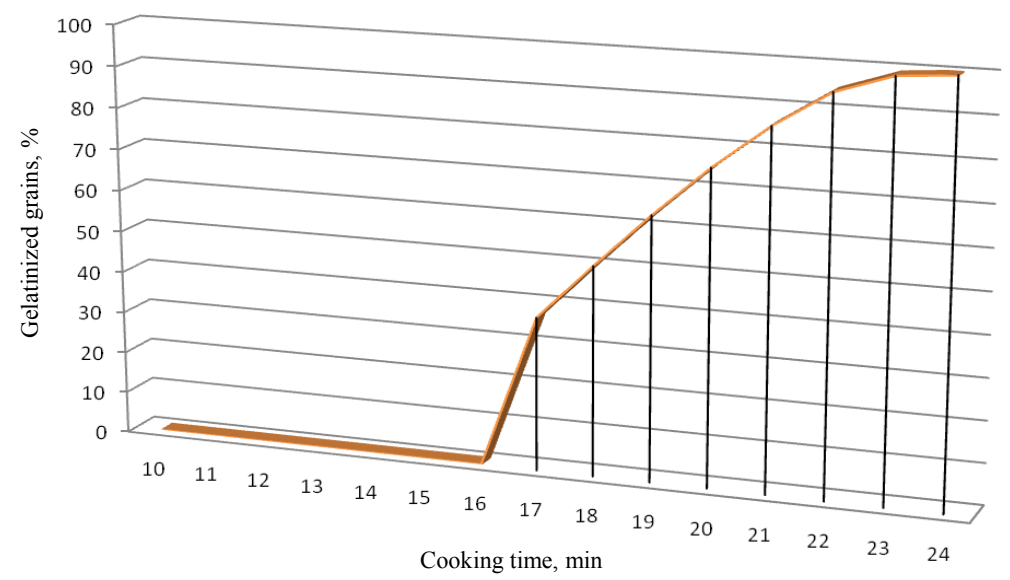

Figure 3. Gelatinization profile of HD6 rice samples. 


\section{Flavor volatiles in the three iranian rice cultivars}

Using headspace SPME method, flavor volatiles in the three Iranian rices were extracted during gelatinization and analyzed by GC-MS. These compounds were determined by comparing their mass spectra and RI values with authentic compound, whereas others were identified by their corresponding mass spectra (Adams mass libraries) and RI values, when RI values on the HP-5MS capillary column were available in the literature ${ }^{13,14}$. A whole range of 74, 55 and 66 components were identified for Hashemi, HD5 and HD6, respectively. For HD5, 23, 11, 23 and 36 specific compounds were identified at stages I, II, III and IV, respectively. And regarding HD6, 14, 19, 47 and 32 specific compounds were identified at the same stages as HD5 and 14, 42, 37 and 43 particular compounds for Hashemi.

In general, the volatile compounds in the three Iranian rice samples during gelatinization, belong to the chemical classes of aldehydes, ketones, hydrocarbons, esters and phenols, etc. these chemical classes corresponding with those previously mentioned in non- Iranian rice using various methods of extraction.

Table 1, 2 and 3 show all compounds detected in the collected rice samples. Those compounds which are listed in Table 1, 2 and 3 were mentioned before in rice. However, 56 new unique compounds were detected (Docosane, 2-Methyl-undecanal, Phthalic acid, Tetracosane, 1,3-dimethoxy-Benzene, Anizole, Pentacosane, Heneicosane, Tricosane, Octane, Octyl formate, 2,6- Dimethoxy-phenol, Neryl acetone, Tetradecanal, 1-Methoxynaphthalene, 2-acetylnaphthalene, Naphthalene, 1-Hexadecene, ar-Turmerone, 2-Ethylhexyl-salicylate, 1,3Dimethoxy-benzene, Biphenyl, Isopropyl tetradecanoate, $<2 \mathrm{Z}, 6 \mathrm{E}>$ Dodecadiene-1-al, alphaPinene, Camphene, $<6$-isopropyl $>$ Quinoline, $<2$-acetyl $>$ Furan, 1,2-Benzenedicarboxylic acid, n- Octyl- 1- iodo- Octane, 2- butyl-2- Octenal, 2, 6, 10, 14- tetramethyl- Hexadecane, 2hydroxy, 2-methyl- Benzoic acid, beta.Bisabolene, 2-Methyl-naphthalene, Cyclosativene, 1,4Dimethylnaphthalene, Methyl isoeugenol, Diethylphthalate, Isobutyl salicylate, Turmerone, <6-methyl->Heptan-2-ol, 1,3-Diethyl-benzene, 1,2,4-Trimethyl benzene, lilia, 2,3,6Trimethyl-naphthalene, 1- ethyl- Naphthalene, 3-methyl-Tetradecane, 3-methyl-Pentadecane, 2,6,10,14-tetramethyl-Pentadecane, 1,3- dimethyl- Naphthalene, 2- phenyl- 2- methylAziridine, 2- methyl- Tridecane, 4-ethyl- 3,4- dimethyl- Cyclohexanone, n- OctylCyclohexane, 9- methyl- Nonadecane), which were not reported in the previous studies ${ }^{15,16}$.

Table 1. Flavor volatiles identified in headspace of Hashemi rice cultivar.

\begin{tabular}{|c|c|c|c|c|c|c|c|c|}
\hline NO & Compound & $\begin{array}{c}\text { Odor } \\
\text { Description } \\
\end{array}$ & $\begin{array}{c}\mathrm{KI} \text { in } \\
\text { experiment }\end{array}$ & $\begin{array}{c}\mathrm{KI} \text { in } \\
\text { literature }\end{array}$ & $\begin{array}{l}\mathrm{I}, \\
\%\end{array}$ & $\begin{array}{l}\mathrm{II}, \\
\%\end{array}$ & $\begin{array}{c}\text { III, } \\
\%\end{array}$ & $\begin{array}{c}\mathrm{IV}, \\
\%\end{array}$ \\
\hline 1 & $N$-Hexanal & \multirow[t]{4}{*}{$\begin{array}{c}\text { Green, } \\
\text { grass-like }\end{array}$} & 805 & 802 & 31.2 & 3.8 & \multirow[t]{3}{*}{7.4} & 12.4 \\
\hline 2 & 2-Butylfuran & & 881 & 890 & 4.3 & & & \\
\hline 3 & $\begin{array}{l}\text { 2- Phenyl-2- } \\
\text { methyl- Aziridine }\end{array}$ & & 888 & & & & & 0.6 \\
\hline 4 & $\begin{array}{l}\text { 2-Acetyl-1- } \\
\text { pyrroline }\end{array}$ & & 915 & 920 & & & \multirow[t]{3}{*}{3.7} & 5.8 \\
\hline 5 & Benzaldehyde & Nutty, bitter & 960 & 960 & & 2.1 & & 1.3 \\
\hline \multirow[t]{2}{*}{6} & $\begin{array}{l}<6 \text {-Methyl- }> \\
\text { Heptan-2-ol }\end{array}$ & & 960 & 965 & & 1.5 & & \\
\hline & & & & & & & \multicolumn{2}{|c|}{ Condt... } \\
\hline
\end{tabular}




\begin{tabular}{|c|c|c|c|c|c|c|c|c|}
\hline 7 & 1-Octen-3-ol & $\begin{array}{c}\text { Raw } \\
\text { mushroom }\end{array}$ & 982 & 979 & & & & 0.9 \\
\hline 8 & 2-Pentylfuran & Nutty, bean & 998 & 990 & & 1.2 & 1.6 & 3.2 \\
\hline 9 & 1,2,4-Trimethyl benzene & & 999 & 994 & & 0.8 & & 0.3 \\
\hline 10 & Octanal & $\begin{array}{l}\text { Green, } \\
\text { citrus- } \\
\text { like }\end{array}$ & 1007 & 999 & 2.1 & 0.9 & 1.6 & 3.5 \\
\hline 11 & D- Limonene & & 1042 & 1029 & & & & 1.3 \\
\hline 12 & 1-Nitro-hexane & & 1051 & & & 2.0 & 2.3 & 2.4 \\
\hline 13 & 1.3-Diethyl-benzene & & 1065 & 1059 & & 1.2 & & \\
\hline 14 & $N$-Octanol & & 1072 & 1068 & & & & 1.8 \\
\hline 15 & $N$-Nonanal & $\begin{array}{l}\text { Soapy, } \\
\text { citrus- } \\
\text { like }\end{array}$ & 1110 & 1101 & 5.9 & 6.0 & 7.7 & 10.4 \\
\hline 16 & (E)-2-Nonenal & $\begin{array}{l}\text { Fatty, } \\
\text { tallowy }\end{array}$ & 1166 & 1162 & & & 1.2 & 0.6 \\
\hline 17 & Naphthalene & & 1173 & 1181 & & 2.2 & & \\
\hline 18 & 2,6-Dimethylaniline & & 1179 & 1168 & & 0.5 & & \\
\hline 19 & Dodecane & & 1201 & 1200 & & & 1.4 & 1.6 \\
\hline 20 & Decanal & $\begin{array}{l}\text { Green, } \\
\text { soapy }\end{array}$ & 1207 & 1207 & & & 2.7 & 2.4 \\
\hline 21 & (E,E)-2,4-Nonadienal & Fatty, waxy & 1207 & 1212 & & 1.8 & & \\
\hline 22 & (E,E)2,4-Octadienal & & 1223 & & & & 0.8 & 1.5 \\
\hline 23 & (E)-2-Decenal & waxy & 1270 & 1264 & & 5.9 & & 2.0 \\
\hline 24 & (E,Z)-2,4-Decadienal & Fatty, green & 1283 & 1293 & & 6.8 & & \\
\hline 25 & Indole & $\begin{array}{l}\text { Sweet, } \\
\text { burnt, floral }\end{array}$ & 1289 & 1291 & & 0.4 & & \\
\hline 26 & $\begin{array}{c}<\text { cis-2-tert-butyl- } \\
>\text { Cyclohexanol acetate }\end{array}$ & & 1302 & 1293 & & 1.2 & 1.4 & 0.6 \\
\hline 27 & Tridecane & & 1303 & 1300 & 1.6 & & 3.3 & 3.4 \\
\hline 28 & (E,E)-2,4-Decadienal & Fatty, waxy & 1310 & 1317 & & 3.0 & 3.3 & \\
\hline 29 & 2-Methyl-naphthalene & & 1324 & & & 1.2 & 1.3 & 0.3 \\
\hline 30 & 1-Methoxy-naphthalene & & 1343 & 1346 & & 1.1 & & 0.7 \\
\hline 31 & 2,6-Dimethoxy-phenol & & 1355 & 1349 & & 3.3 & & \\
\hline 32 & 2- methyl- Tridecane & & 1366 & & & & & 0.4 \\
\hline 33 & Cyclosativene & & 1371 & 1383 & & & & 0.4 \\
\hline 34 & Undecanol & & 1372 & 1370 & & & 0.6 & 1.9 \\
\hline 35 & $\begin{array}{c}\text { 2,6,10,14-tetramethyl- } \\
\text { Hexadecane }\end{array}$ & & 1379 & & & & 2.5 & 3.1 \\
\hline 36 & Tetradecane & & 1402 & 1400 & 3.7 & 4.8 & 7.8 & 6.9 \\
\hline 37 & Cyclohexanone & & 1405 & & & & & 0.4 \\
\hline 38 & (E)-2-Octenal & Green, fatty & 1420 & 1425 & & 1.7 & 1.5 & 1.3 \\
\hline 39 & 2,6-Dimethylnaphthalene & & 1427 & 1431 & & 2.6 & 1.0 & 1.4 \\
\hline 40 & Isobutyl salicylate & & 1429 & 1425 & 3.1 & 2.1 & & \\
\hline 41 & 1,3- dimethyl- Naphthalene & & 1434 & & & 1.3 & & \\
\hline 42 & 2-Pentadecanone & & 1441 & & 9.2 & & & \\
\hline 43 & 1,4-Dimethylnaphthalene & & 1450 & & & 4.2 & 5.1 & 0.8 \\
\hline 44 & n- Octyl-Cyclohexane & & 1459 & & & & & 1.0 \\
\hline 45 & Isoeugenol & & 1461 & 1451 & & & & $\begin{array}{r}0.5 \\
n d t \ldots\end{array}$ \\
\hline
\end{tabular}




\begin{tabular}{|c|c|c|c|c|c|c|c|c|}
\hline 46 & 9- Methyl- Nonadecane & & 1463 & & & & & 1.6 \\
\hline 47 & Geranyl acetone & $\begin{array}{l}\text { Magnolia, } \\
\text { green }\end{array}$ & 1464 & 1455 & & 2.9 & & \\
\hline 48 & $<2$ E- $>$ Dodecenal & & 1468 & 1466 & & & 4.2 & \\
\hline 49 & 3-Methyl-Tetradecane & & 1472 & & & & 2.4 & 0.4 \\
\hline 50 & (E,E)-2,4-Heptadienal & fatty, hay-like & 1485 & & & 1.2 & & \\
\hline 51 & 1- Ethyl- Naphthalene & & 1487 & & & 1.0 & & \\
\hline 52 & Pentadecane & & 1499 & 1500 & 1.8 & 2.7 & 3.1 & 3.3 \\
\hline 53 & N.D. & & 1516 & & & & 1.3 & \\
\hline 54 & Beta.Bisabolene & & 1525 & 1506 & & & 1.0 & 0.5 \\
\hline 55 & lilial & & 1535 & 1529 & & 1.2 & & \\
\hline 56 & N.D. & & 1548 & & & & 1.4 & \\
\hline 57 & Diethylphthalate & & 1556 & 1565 & 5.7 & & & \\
\hline 58 & 1-Octanol & Fruity, floral & 1560 & 1565 & & 1.7 & 1.6 & \\
\hline 59 & 2,3,6-Trimethyl-naphthalene & & 1565 & & & 1.7 & 1.8 & \\
\hline 60 & 3-Methyl-Pentadecane & & 1573 & & & & 0.9 & 2.2 \\
\hline 61 & 2,3,5-Trimethyl-naphthalene & & 1582 & & & 1.0 & 2.2 & \\
\hline 62 & Hexadecane & & 1602 & 1600 & 7.7 & 4.4 & 5.1 & 2.4 \\
\hline 63 & 2-Acetyl-naphthalene & & 1615 & 1609 & & 2.5 & & \\
\hline 64 & N.D. & & 1628 & & & 0.9 & & \\
\hline 65 & $\begin{array}{l}\text { 2,6,10-Trimethyl- } \\
\text { pentadecane }\end{array}$ & & 1640 & 1644 & & 1.0 & 1.2 & 0.3 \\
\hline 66 & Turmerone & & 1675 & 1669 & & 1.6 & & \\
\hline 67 & Heptadecane & & 1703 & 1700 & & 3.8 & 4.0 & 0.2 \\
\hline 68 & N.D. & & 1704 & & & & 2.3 & \\
\hline 69 & $\begin{array}{c}\text { 2,6,10,14-Tetramethyl- } \\
\text { Pentadecane }\end{array}$ & & 1712 & & & & 1.6 & 1.0 \\
\hline 70 & (E)-2-Undecenal & Fatty, sweet & 1755 & 1750 & & 5.7 & 5.0 & \\
\hline 71 & Octadecane & & 1800 & 1800 & 10.0 & & & \\
\hline 72 & Nonadecane & & 1890 & 1900 & 5.0 & & & 1.6 \\
\hline 73 & 2-Methoxy-4-vinylphenol & $\begin{array}{l}\text { Spicy, } \\
\text { clove-like }\end{array}$ & 2195 & 2180 & & & 2.3 & 4.6 \\
\hline 74 & Tetracosane & & 2409 & 2400 & 7.6 & 3.1 & & \\
\hline Total & & & 805 & 802 & 99.0 & 99.8 & 99.7 & 93.0 \\
\hline
\end{tabular}

Table 2. Flavor volatiles identified in headspace of HD5 rice cultivar.

\begin{tabular}{ccccccccc}
\hline NO & Compound & $\begin{array}{c}\text { Odor } \\
\text { Description }\end{array}$ & $\begin{array}{c}\text { KI in } \\
\text { experiment }\end{array}$ & $\begin{array}{c}\text { KI in } \\
\text { literature }\end{array}$ & $\begin{array}{c}\text { I, } \\
\%\end{array}$ & $\begin{array}{c}\text { II, } \\
\%\end{array}$ & $\begin{array}{c}\text { III, } \\
\%\end{array}$ & $\begin{array}{c}\text { IV, } \\
\%\end{array}$ \\
\hline 1 & $n$-Hexanal & $\begin{array}{c}\text { Green, grass- } \\
\text { like }\end{array}$ & 801 & 802 & 62.3 & 56.7 & 38.9 & 22.2 \\
2 & $n$-Octane & & 809 & 800 & 0.7 & & & \\
3 & $n$-Hexanol & Herbaceous & 869 & 871 & & & & 1.1 \\
4 & $n$-Heptanal & Fruity, fatty & 899 & 902 & 1.9 & & & 3.3 \\
& & & & & & & & \\
& & &
\end{tabular}




\begin{tabular}{|c|c|c|c|c|c|c|c|c|}
\hline 5 & 2- Acetyl-1-pyrroline & & 915 & 920 & 1.4 & & & \\
\hline 6 & Anizole & & 917 & 918 & 1.8 & 3.3 & 3.5 & 3.9 \\
\hline 7 & Ethanol & sweet & 920 & 925 & 0.9 & & & \\
\hline 8 & Benzaldehyde & Nutty, bitter & 974 & 960 & 0.9 & & & \\
\hline 9 & $\begin{array}{c}\text { Hepten-2-ol<6- } \\
\text { methyl-5-> }\end{array}$ & & 982 & 992 & 1.0 & & & \\
\hline 10 & 2-Pentylfuran & Nutty, bean & 985 & 990 & 4.0 & 11.2 & 3.3 & 4.3 \\
\hline 11 & 1-Octen-3-ol & Raw mushroom & 989 & 979 & & & & 2.4 \\
\hline 12 & $\begin{array}{c}\text { 1,3,5-trimethyl- } \\
\text { Benzene }\end{array}$ & & 990 & 996 & 0.8 & 4.8 & & \\
\hline 13 & $n$-Octanal & $\begin{array}{c}\text { Green, citrus- } \\
\text { like }\end{array}$ & 993 & 999 & 2.6 & & 2.7 & 4.4 \\
\hline 14 & $n$-Decane & & 1009 & 1000 & & & 1.6 & 1.7 \\
\hline 15 & $D$ - Limonene & & 1022 & 1029 & & & 6.2 & \\
\hline 16 & Undecane & & 1091 & 1100 & 0.6 & & & \\
\hline 17 & $n$-Nonanal & Soapy, citrus-like & 1100 & 1101 & 6.1 & 7.6 & 8.8 & 11.7 \\
\hline 18 & Octyl formate & & 1140 & 1131 & & & & 1.9 \\
\hline 19 & (E)-2-Nonenal & Fatty, tallowy & 1157 & 1162 & & & & 1.4 \\
\hline 20 & $\begin{array}{l}\text { 1,3-dimethoxy- } \\
\text { Benzene }\end{array}$ & & 1178 & 1169 & 1.2 & & & \\
\hline 21 & Dodecane & & 1200 & 1200 & 2.5 & 1.6 & 0.5 & 1.1 \\
\hline 22 & Tridecane & & 1297 & 1300 & 0.4 & 3.2 & 1.7 & 2.4 \\
\hline 23 & E-2-Heptenal & Herbaceous & 1320 & 1318 & & & & 1.4 \\
\hline 24 & Hexyl furan & & 1322 & 1329 & & & & 2.1 \\
\hline 25 & $\begin{array}{l}\text { 2,6-Dimethoxy- } \\
\text { phenol }\end{array}$ & & 1340 & 1349 & & & & 3.5 \\
\hline 26 & 2- Methyl- undecanal & & 1366 & 1368 & & & & 0.9 \\
\hline 27 & $n$ - Undecanol & & 1372 & 1370 & & & & 0.8 \\
\hline 28 & N.D. & & 1379 & & & & 0.9 & \\
\hline 29 & N.D. & & 1379 & & & & & 0.7 \\
\hline 31 & Tetradecane & & 1391 & 1400 & & 4.0 & 4.1 & 3.0 \\
\hline 32 & (E)-2-Octenal & Green,fatty & 1432 & 1425 & & & & 2.6 \\
\hline 33 & $\begin{array}{l}\text { 2,6-Dimethyl- } \\
\text { naphthalene }\end{array}$ & & 1442 & 1431 & & & & 1.3 \\
\hline 34 & Geranyl acetone & Magnolia, green & 1463 & 1455 & & & & 1.6 \\
\hline 35 & 1-Heptanol & Green,fatty & 1466 & 1457 & & & 1.6 & \\
\hline 36 & N.D. & & 1469 & & & & & 1.7 \\
\hline 37 & N.D. & & 1491 & & 0.4 & & & \\
\hline 38 & Pentadecane & & 1500 & 1500 & & 3.6 & 6.3 & 3.6 \\
\hline 39 & $\begin{array}{c}\text { Butylated } \\
\text { hydroxytoluene }\end{array}$ & & 1524 & 1516 & & & & 1.5 \\
\hline 40 & 1-Octanol & Fruity, floral & 1560 & 1565 & 0.6 & & 1.0 & \\
\hline 41 & Hexadecane & & 1600 & 1600 & & 2.2 & 4.8 & 2.1 \\
\hline 42 & 1-Nonanol & Floral, citrus-like & 1670 & 1671 & & & & 1.1 \\
\hline 43 & $n$-Tetradecanol & & 1670 & 1673 & & & & 1.0 \\
\hline 44 & Heptadecane & & 1700 & 1700 & & & 0.6 & 1.0 \\
\hline 45 & Phthalic acid & & 1703 & & 3.4 & & & \\
\hline 46 & Farnesol & & 1710 & 1718 & 2.2 & & & \\
\hline 47 & 1-Octadecene & & 1799 & 1790 & & & & 0.6 \\
\hline 48 & n-Nonadecane & & 1905 & 1900 & & & & 0.7 \\
\hline \multicolumn{9}{|c|}{ Condt... } \\
\hline
\end{tabular}




\begin{tabular}{cccccccc}
\hline 49 & n-Nonadecane & 1907 & 1900 & & & 4.1 & \\
50 & n-Eicosane & 1995 & 2000 & 0.3 & & 2.0 & \\
51 & n-Heneicosane & 2101 & 2100 & & 1.9 & 0.7 & 1.2 \\
52 & Docosane & 2209 & 2200 & 0.5 & & 0.7 & 3.5 \\
53 & n-Tricosane & 2291 & 2300 & & & 2.0 & \\
54 & Tetracosane & 2411 & 2400 & 0.6 & & 2.2 & 0.6 \\
55 & Pentacosane & 2510 & 2500 & & & 1.8 & 1.3 \\
\hline Total & & & 97.0 & 100.0 & 99.9 & 99.3 \\
\hline
\end{tabular}

Table 3. Flavor volatiles identified in the headspace of HD6 rice cultivar.

\begin{tabular}{|c|c|c|c|c|c|c|c|c|}
\hline NO & Compound & Odor Description & $\begin{array}{c}\text { KI in } \\
\text { experiment }\end{array}$ & $\begin{array}{c}\mathrm{KI} \text { in } \\
\text { literature }\end{array}$ & $\begin{array}{l}\mathrm{I}, \\
\%\end{array}$ & $\begin{array}{l}\mathrm{II}, \\
\%\end{array}$ & $\begin{array}{l}\text { III, } \\
\%\end{array}$ & $\begin{array}{l}\mathrm{IV}, \\
\%\end{array}$ \\
\hline 1 & Pentanal & Woody, fruity & 710 & 706 & & & 1.5 & \\
\hline 2 & Octane & & 801 & 800 & & & 1.4 & \\
\hline 3 & $N$-Hexanal & Green, grass-like & 802 & 802 & 49.0 & 42.2 & 14.2 & 36.3 \\
\hline 4 & $N$-Heptanal & Fruity, fatty & 900 & 902 & 3.0 & 2.2 & & 2.2 \\
\hline 5 & $<2$-Acetyl $>$ Furan & & 911 & 913 & & & & 1.8 \\
\hline 6 & Anizole & & 919 & 918 & 5.3 & 7.1 & 5.2 & 2.5 \\
\hline 7 & Alpha-Pinene & & 935 & 939 & & & & 1.5 \\
\hline 8 & Camphene & & 951 & 954 & & & & 1.1 \\
\hline 9 & Benzaldehyde & Nutty, bitter & 960 & 960 & & & 2.0 & 1.2 \\
\hline 10 & 2-Pentylfuran & Nutty, bean & 981 & 990 & 6.6 & 4.4 & 3.8 & 5.3 \\
\hline 11 & 1-Octen-3-ol & Raw mushroom & 982 & 979 & 1.9 & 2.0 & 2.2 & 1.3 \\
\hline 12 & Octanal & Green, citrus-like & 990 & 999 & & 4.7 & 2.3 & \\
\hline 13 & $\begin{array}{c}\text { 1,3,5-trimethyl- } \\
\text { Benzene }\end{array}$ & & 992 & 996 & 4.0 & & & 2.8 \\
\hline 14 & $D$ - Limonene & & 1020 & 1029 & & & & 2.6 \\
\hline 15 & Undecane & & 1091 & 1100 & & & 2.7 & \\
\hline 16 & $N$-Nonanal & Soapy, citrus-like & 1095 & 1101 & 10.9 & 17.4 & 10.0 & 6.1 \\
\hline 17 & Octyl formate & & 1139 & 1131 & & 1.7 & & 0.9 \\
\hline 18 & (E)-2-Nonenal & Fatty, tallowy & 1152 & 1162 & & 0.9 & & 0.5 \\
\hline 19 & $\begin{array}{l}\text { 1,3-Dimethoxy- } \\
\text { benzene }\end{array}$ & & 1175 & 1169 & & & 0.4 & 1.1 \\
\hline 20 & Naphthalene & & 1187 & 1181 & & & 1.0 & \\
\hline 21 & Dodecane & & 1200 & 1200 & & & 0.3 & \\
\hline 22 & $\begin{array}{c}n \text { - Octyl- } 1 \text { - iodo- } \\
\text { Octane }\end{array}$ & & 1250 & & & 2.6 & & \\
\hline 23 & $(E)$-2-Decenal & waxy & 1260 & 1264 & & & 1.2 & \\
\hline 24 & $(E, Z)-2,4$-Decadienal & Fatty, green & 1299 & 1293 & & & 1.3 & \\
\hline 25 & Tridecane & & 1300 & 1300 & 1.4 & 1.1 & 1.4 & 0.9 \\
\hline 26 & Undecanal & Fresh, lemon-like & 1312 & 1307 & & 0.6 & 0.3 & \\
\hline 27 & (E)-2-Heptenal & Herbaceous & 1316 & 1318 & & & 0.8 & 0.7 \\
\hline 28 & $\begin{array}{c}2,6- \\
\text { Dimethoxyphenol }\end{array}$ & & 1357 & 1349 & 0.9 & 1.8 & 4.6 & \\
\hline 29 & 2- Butyl-2- Octenal & & 1383 & & & 0.7 & 1.0 & \\
\hline 30 & Biphenyl & & 1385 & 1377 & & & 0.6 & \\
\hline 31 & $\begin{array}{l}\text { Tetradecane } \\
2,6,10,14-\end{array}$ & & 1400 & 1400 & 2.2 & 2.3 & 2.1 & 1.6 \\
\hline 32 & $\begin{array}{l}\text { tetramethyl- } \\
\text { Hexadecane }\end{array}$ & & 1421 & & & & & 3.0 \\
\hline 33 & (E)-2-Octenal & Green, fatty & 1433 & 1425 & & 1.4 & 1.7 & 1.7 \\
\hline
\end{tabular}




\begin{tabular}{|c|c|c|c|c|c|c|c|c|}
\hline 34 & $\begin{array}{c}2,6- \\
\text { Dimethylnaphthalene }\end{array}$ & & 1437 & 1431 & & & 1.2 & \\
\hline 35 & $\begin{array}{l}\text { 1-Methoxy- } \\
\text { naphthalene }\end{array}$ & & 1440 & 1446 & & & 1.2 & \\
\hline 36 & Neryl acetone & & 1441 & 1436 & & 2.1 & 1.0 & 1.0 \\
\hline 37 & $\begin{array}{l}\text { 2- hydroxy, 2- } \\
\text { methyl- Benzoic acid }\end{array}$ & & 1449 & & & & & 2.3 \\
\hline 38 & $\begin{array}{c}<2 \mathrm{Z}, 6 \mathrm{E}> \\
\text { Dodecadiene-1-al }\end{array}$ & & 1455 & 1447 & & & 0.5 & \\
\hline 39 & 2-Pentadecanone & & 1459 & 1451 & & & 7.5 & \\
\hline 40 & Geranyl acetone & Magnolia, green & 1460 & 1455 & 1.4 & & 2.2 & \\
\hline 41 & $\begin{array}{l}\text { 1,2- Benzenedi- } \\
\text { carboxylic acid }\end{array}$ & & 1469 & & 2.4 & & & \\
\hline 42 & Pentadecane & & 1500 & 1500 & 2.1 & 2.6 & 2.7 & 1.3 \\
\hline 43 & $\begin{array}{c}<6 \text {-isopropyl }> \\
\text { Quinoline }\end{array}$ & & 1519 & 1511 & & & & 0.9 \\
\hline 44 & N.D. & & 1539 & & & & 0.6 & \\
\hline 45 & N.D. & & 1553 & & & & & 2.7 \\
\hline 46 & 1-Octanol & Fruity, floral & 1570 & 1565 & & & 1.3 & \\
\hline 47 & N.D. & & 1573 & & & & 0.6 & \\
\hline 48 & N.D. & & 1595 & & & & & 2.0 \\
\hline 49 & 1-Hexadecene & & 1599 & 1590 & & & 0.7 & \\
\hline 50 & Hexadecane & & 1600 & 1600 & 2.1 & 2.4 & 4.4 & 4.0 \\
\hline 51 & 2-acetylnaphthalene & & 1602 & 1609 & & & 1.6 & \\
\hline 52 & N.D. & & 1612 & & & & 0.8 & \\
\hline 53 & Tetradecanal & & 1617 & 1613 & & & 0.6 & \\
\hline 54 & N.D. & & 1658 & & & & 0.7 & \\
\hline 55 & N.D. & & 1673 & & & & 0.6 & \\
\hline 56 & ar-Turmerone & & 1675 & 1669 & & & 1.7 & \\
\hline 57 & 1-Nonanol & Floral, citrus-like & 1681 & 1671 & & & 0.7 & \\
\hline 58 & Heptadecane & & 1700 & 1700 & & & & 0.9 \\
\hline 59 & (E)-2-Undecenal & Fatty, sweet & 1759 & 1750 & & & 0.9 & \\
\hline 60 & Octadecane & & 1809 & 1800 & & & & 3.1 \\
\hline 61 & $\begin{array}{l}\text { 2-Ethylhexyl- } \\
\text { salicylate }\end{array}$ & & 1811 & 1807 & & & 1.8 & \\
\hline 62 & Tridecanal & & 1830 & 1821 & & & & 0.7 \\
\hline 63 & $\begin{array}{l}\text { Isopropyl } \\
\text { tetradecanoate }\end{array}$ & & 1839 & 1830 & & & 1.3 & 2.1 \\
\hline 64 & Hexadecanoic acid & & 2006 & 1995 & & & & 2.7 \\
\hline 65 & $\begin{array}{l}\text { 2-Methoxy-4- } \\
\text { vinylphenol }\end{array}$ & Spicy,clove-like & 2191 & 2180 & & & 1.0 & \\
\hline 66 & Docosane & & 2209 & 2200 & & & 1.0 & \\
\hline Total & & & & & 93 & 100 & 92.3 & 98.7 \\
\hline
\end{tabular}

Variation in flavor volatiles of rice during four different cooking stages

Significant differences were investigated in the volatiles of rice during the four different gelatinization stages. Two major compounds were detected at stage I for Hashemi, HD5 and HD6 rice cultivars known as nonanal and hexanal. The latter is known as an important lipid oxidation product in rice. However, there are other components which have been identified only at stage I for HD5, such as ethanol, which were apparently lost by steam vaporization at later cooking stages during the cooking process. In contrast, hexadecanoic acid which was 
identified as a predominant compound at stage IV for HD6, as well as pentacosane were detected at stage III \& IV for HD5.

Primary heating of rice at gelatinization stages I and II resulted in the evaporation of aldehydes in rice, and fatty acids in steam distillation in all cultivars, respectively. But, excess steam and heat have debilitative influence on the extraction of low-boiling-point volatiles. Thus, further heating at stages III and IV, increased the rate of evaporation of a broad range of the flavor volatiles of rice. As anticipated, the amount of key odorant compounds for instance (E)-2 nonenal (HD5, HD6 and Hashemi), (E,Z)-2, 4-decadienal (HD6 and Hashemi), 2-methoxy-4-vinylphenol (HD6 and Hashemi) and indole (Hashemi) commonly increased, while those of low-boiling point volatiles decreased upon cooking in all cultivars.

\section{Similarities and differences among the three different Iranian rice cultivars}

By comparing the volatile components in Table 1, 2 and 3 it might infer the similarities and differences in the three Iranian rice samples. These samples were examined on the basis of the same condition.

There were no significant differences in the profiles of flavor volatiles; nevertheless, less volatile compounds were recognized in two modified rice samples (HD5 \& HD6), comparing Hashemi. For instance, some components such as Phthalic acid, Farnesol, Ethanol, alpha-Pinene and Camphene were detected in two modified samples, whereas Hashemi's major compounds were beta.Bisabolene, Cyclosativene, Methyl isoeugenol, Isobutyl salicylate, Turmerone, lilia, <cis-2-tert-butyl->Cyclohexanol acetate, 2-phenyl- 2methyl- Aziridine, 4-ethyl- 3,4- dimethyl- Cyclohexanone, n- Octyl- Cyclohexane, 9methyl- Nonadecane, 2- methyl- Tridecane, $(E, E)-2,4-$ Decadienal and Indole.

A broad range of the flavor volatiles of rice during gelatinization could be extracted and identified in a single headspace SPME/GC-MS, of which Naphthalene compounds and Benzene compounds liberated widely and predominantly from different rice samples, which these components have not been previously reported in rice.

From more than 300 rice volatiles identified by many research groups, 2-acetyl-1pyrroline (2AP) as a principal aroma compound. We were able to detect 2-acetyl-1-pyrroline in the Hashemi rice sample at gelatinization stage III and IV and HD5 rice sample at gelatinization stage I, although we were unable to detect any 2-acetyl-1-pyrroline in the HD6 modified rice sample.

Aromatic can be directly linked to consumer preference in both positive terms (such as 2AP) and negative terms of which Hexanal and 2-Pentylfuran were detected in the headspace of different rice sample.

In Hashemi cultivar the amount of Hexanal decreased significantly at gelatinization stage II and increased gradually at gelatinization stage III and IV. In contrast, the amount of 2-Pentylfuran increased at gelatinization stage II, III and IV.

In HD5 cultivar the amount of Hexanal decreased with increasing gelatinization. The amount of 2-Pentylfuran increased significantly at gelatinization stage II and decreased at stage III, and then increased slightly at stage IV.

In HD6 cultivar the amount of Hexanal decreased with increasing gelatinization stage I, II, III and then increased significantly at gelatinization stage IV. Similar results were observed for the 2-Pentylfuran.

\section{Optimization of different experimental parameters}

In order to optimize the suitable conditions of flavor compounds, hexanal target were investigate. Initially, three types of commercial fibers (PDMS, CAR/PDMS and DVB/CAR/ PDMS) were selected in order to extract volatiles from the headspace above the rice samples 
at the beginning of the experiment. The latter is known as the most effective compound in extraction of flavor volatiles. The DVB/CAR/PDMS fiber was used in all applications (Figure 4).

In order to estimate the effect of water content of rice samples on the SPME of target analytes, different volumes of water were added to $150 \mathrm{~g}$ rice sample and SPME experiments were performed. The results in Figure 1 indicate that increasing water content up to $400 \mathrm{~mL}$ was correspondent with the extraction efficiency increase of hexanal. However, addition of $>400 \mathrm{~mL}$ water showed a great decrease in extraction efficiency, since this would result in producing an adhesive mixture that was not easily agitated and made the diffusion of analytes more difficult. On the other hand, addition of $<400 \mathrm{~mL}$ water was not enough for the cooking of rice. Therefore, $400 \mathrm{~mL}$ water was selected as a suitable amount of water for the rest of experiment (Figure 5).

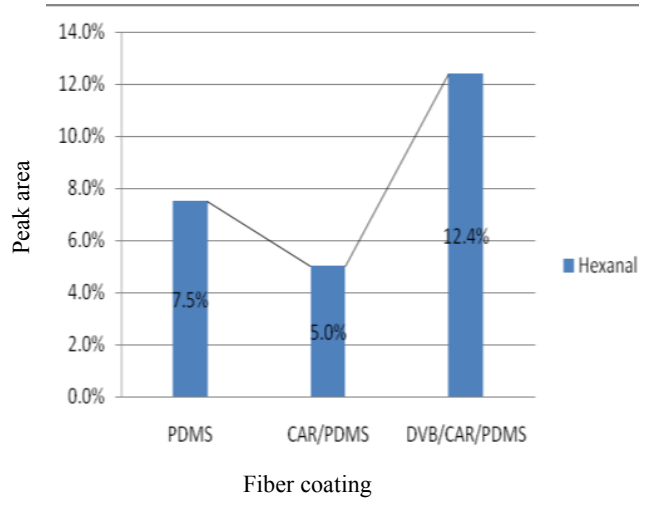

Figure 4. Comparison of the extraction efficiencies of the PDMS, CAR/PDMS and $\mathrm{DVB} / \mathrm{CAR} / \mathrm{PDMS}$ fibers for volatile compounds in the headspace of rice sample.

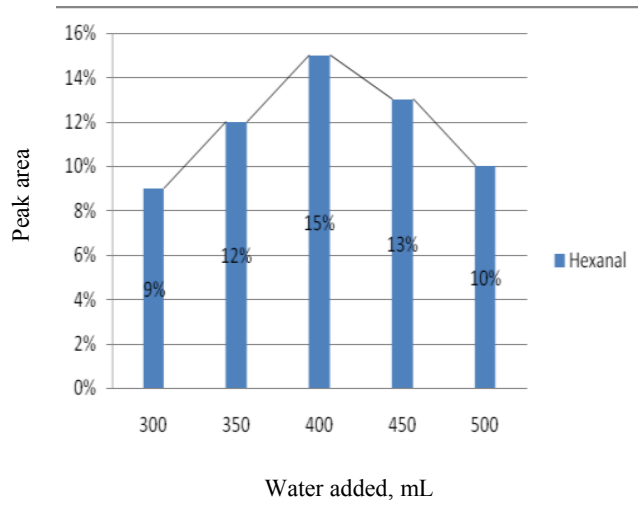

Figure 5. Comparison of the extraction efficiencies of the various additions of water.

For the investigation of the effect of equilibrium time on the target analytes, the rice samples were extracted 5-30 min. As Figure 6 shows, with the increase in equilibrium time, the extracted amounts of hexanal increased in to a maximum threshold after $30 \mathrm{~min}$ of extraction. As a whole, the optimum equilibrium time was considered as $30 \mathrm{~min}$ to reach the equilibrium for hexanal (Figure 6).

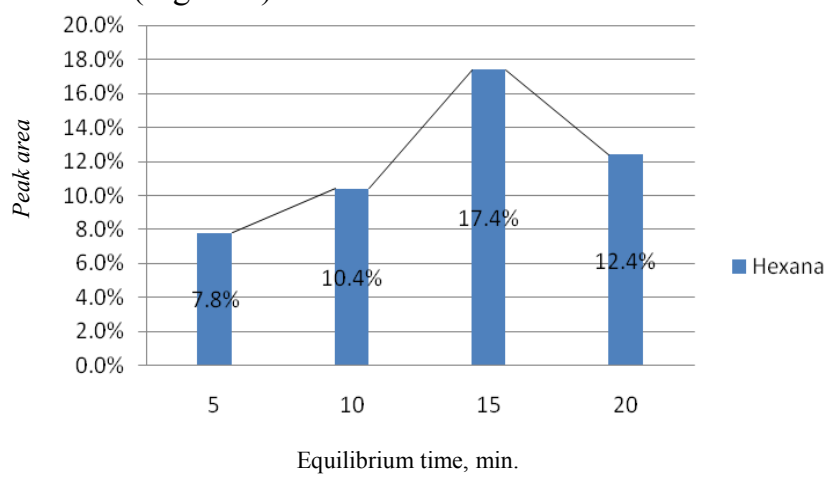

Figure 6. Equilibrium time profile. 


\section{Identification of contact allergens in rice cultivars}

Rice flavor consists of a wide range of volatile compounds. Along with, in this study seven fragrance chemicals were detected, which were most frequently reported as contact allergens. Their names are listed as follows: Lilial (Lilial is a synthetic compound listed as a fragrance allergen), Cyclohexanone, Diethylphthalate, Farnesol (Farnesol is one of the most frequent contact allergens), Isoeugenol (common allergen), Limonene, Alpha-pinene.

\section{Conclusion}

This study has demonstrated the application of HS-SPME/GC-MS as a powerful combination to perform a rapid analytical method for the direct profiling of the flavor volatiles in three different rice cultivars including two modified Iranian rice cultivars and Hashemi rice cultivar during gelatinization process. Therefore, a broad range of the flavor volatiles of rice could be extracted, concentrated and identified in the headspace of different rice samples using HS-SPME/GC-MS.

All of free flavor volatiles, the bound flavor components and the compounds formed by the thermal decomposition of the non-volatile constituents existing in rice should be liberated during the gelatinization process.

This is a first study which was accomplished regarding the contact allergens in rice during gelatinization process, which was not previously reported. Further studies should be accomplished to consider future separation and identification on these compounds, as well as comparing these compounds among Iranian, non- Iranian samples and modified samples.

\section{Acknowledgment}

The authors are so grateful to the Laboratory Complex of I.A.U. for valuable technical assistance.

\section{References}

1. Laohakunjit N and Kerdchoechuen O, Food Chem., 2007, 101, 339-344.

2. Fitzgerald M A, Couch S R M and Hall R D, Trends Plant Sci., 2009, 14, 133-139.

3. Bergman C J, Delgado J T, Bryant R, Grimm C, Cadwallader K R and Webb B D, Cereal Chem., 2000, 77(4), 454-458.

4. Lam H S and Proctor A, J Food Sci., 2003, 68, 2676-2681.

5. Buttery R G, Ling L C and Turnbaugh J G, J Agr Food Chem., 1988, 36, 1006-1009.

6. $\quad$ Paule C M and Powers J, J Food Sci., 1989, 54, 343-347.

7. Laksanalamai V and Ilangantileke S, Cereal Chem., 1993, 70, 381-384.

8. Jezussek M, Juliano B O and Schieberle P, J Agr Food Chem., 2002, 50, 1101-1105.

9. Mehdinia A, Mousavi M F and Shamsipur M, J Chromatogr A, 2006, 1134, 24-31.

10. Djozan D, Makham M and Ebrahimi B, J Chromatogr A, 2009, 1216, 2211-2219.

11. Bergman C J, Delgado J T, Bryant R and Grimm C, J Agr Food Chem., 2001, 49, 245-249.

12. Grimm C, Lioyd S W, Godshall M A and Braggins T J, Adv Exp Med Biol., 2004, 542, 167-174.

13. Buttery R G, Orts W J, Takeoka GR and Nam Y, J Agr Food Chem., 1999, 47, 4353-4356.

14. Fan W and Qian M, J Agr Food Chem., 2006, 54, 2695-2704.

15. Widjaja R, craske J D and wootton M, J Sci Food Agric., 1996, 70,151-156.

16. Yang D S, Lee K S, Jeong Y, Kim K and Kays S, Food Chem., 2008, 56, 235-240. 


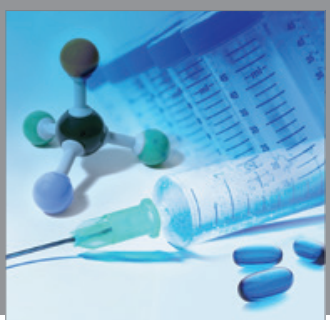

International Journal of

Medicinal Chemistry

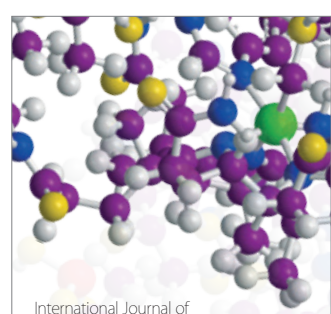

Carbohydrate Chemistry

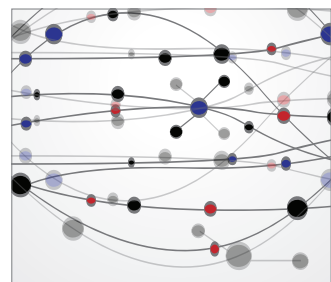

The Scientific World Journal
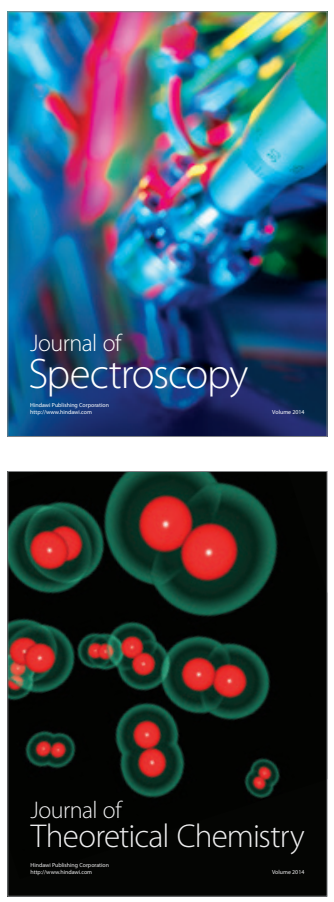
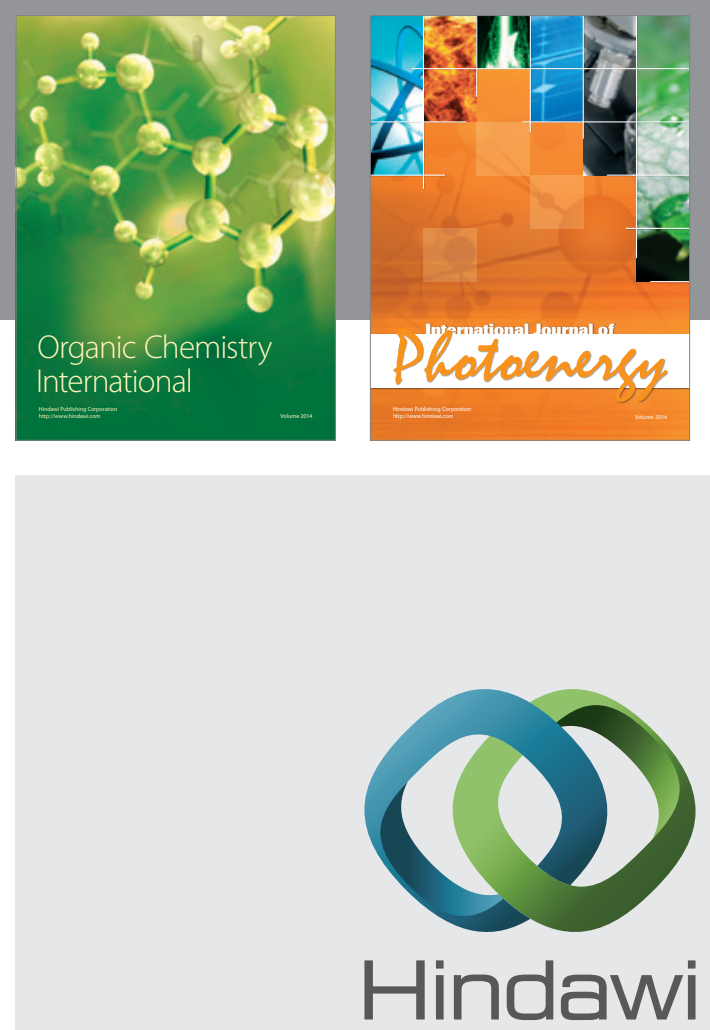

Submit your manuscripts at

http://www.hindawi.com
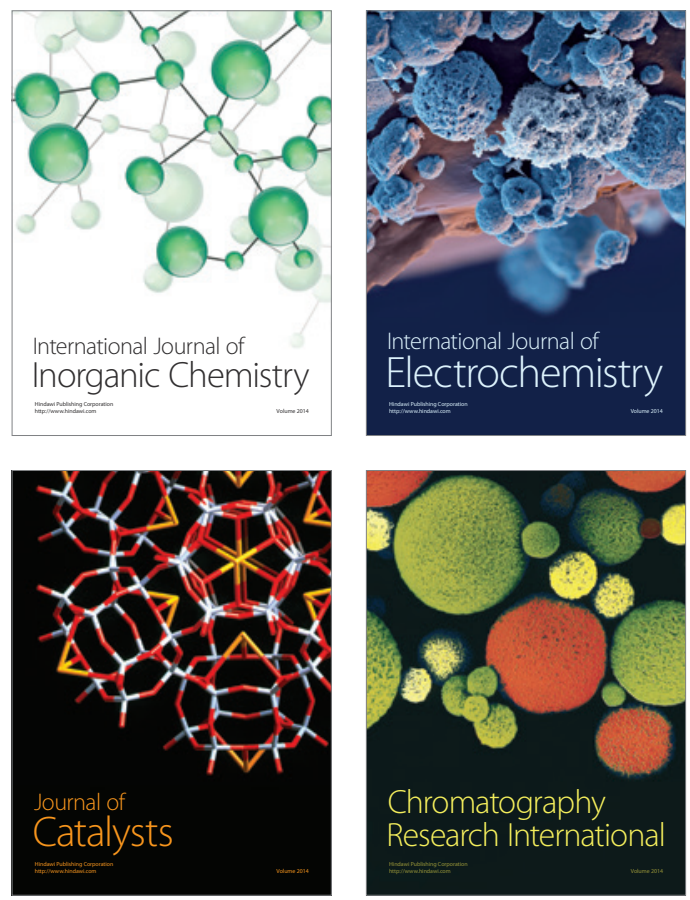
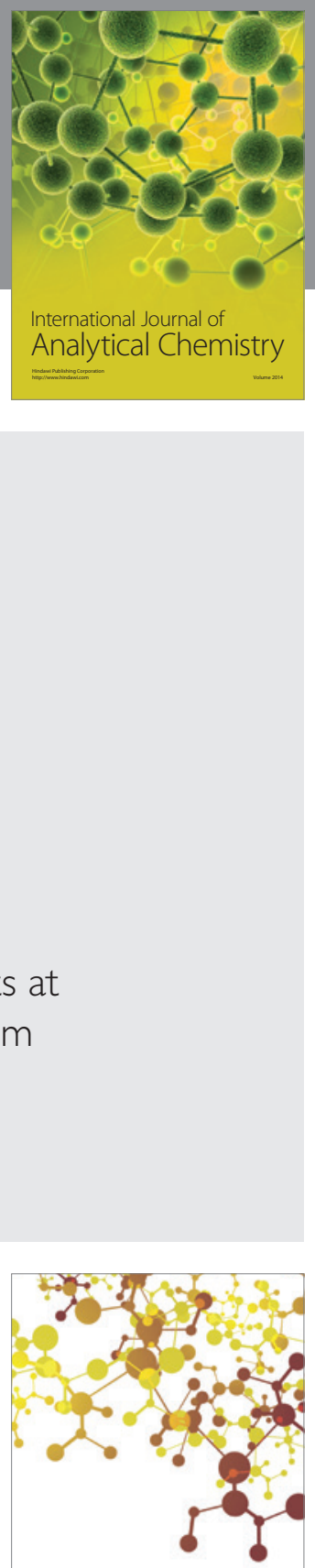

Journal of

Applied Chemistry
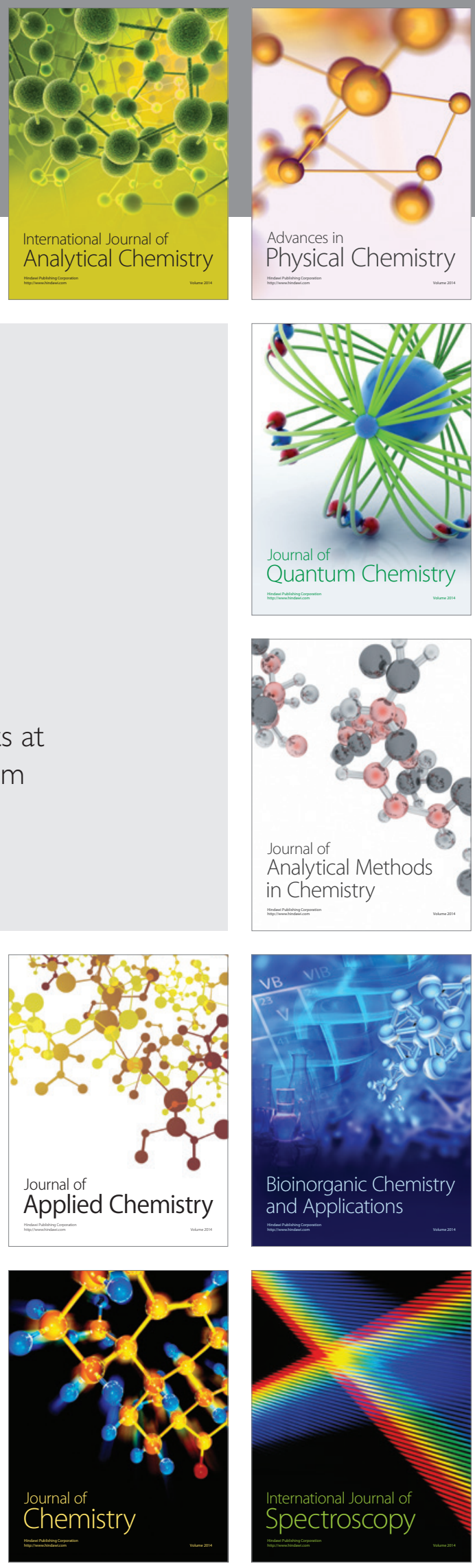